Article

\title{
Development of a Freeze-Drying Stage for In-Situ $\mu-C T$ Measurements
}

\author{
Mathias Hilmer*(D), Sebastian Gruber and Petra Foerst \\ Chair of Process Systems Engineering, TUM School of Life Sciences, Technical University of Munich, \\ Gregor-Mendel-Str. 4, 85354 Freising, Germany; sebi.gruber@tum.de (S.G.); petra.foerst@tum.de (P.F.) \\ * Correspondence: mathias.hilmer@tum.de
}

Received: 29 June 2020; Accepted: 16 July 2020; Published: 18 July 2020

\begin{abstract}
This paper shows the development of a freeze-drying stage for in-situ $\mu$-CT measurements. The stage can operate in a temperature range of $-40^{\circ} \mathrm{C}$ up to $70{ }^{\circ} \mathrm{C}$, and a pressure range from atmospheric pressure to $7 \mathrm{~Pa}$ at the sample holder. To get the best visualization of the probe, it is fundamental that the materials around the sample holder are not absorbing most of the radiation. For this reason, we built an axial symmetrical stage built out of polyetheretherketon (PEEK). A test of the stage by different freeze-drying experiments with maltodextrin and sucrose particles and solutions demonstrated its suitability to visualize the freeze-drying processes in-situ. It was possible to track the drying front during the process by radiographic and tomographic measurements, as well as to visually resolve the ice crystals and porous structure in tomographic measurements. Using different samples and process parameters, we showed that the freeze-drying stage is not only suitable for in-situ $\mu$-CT measurements, but also allows us to use the stage for other imaging methods such as neutron imaging, and for any sample where a controlled environment is needed.
\end{abstract}

Keywords: freeze-drying; in-situ; $\mu$-CT; pore network; sublimation front; $\mathrm{X}$-ray; lyophilization

\section{Introduction}

Freeze-drying is a commonly used method for the preservation of pharmaceutical, biotechnological and food materials. Due to the long drying time and phase transitions, freeze-drying is a time- and cost-intensive process. However, freeze-drying is considered a process that retains most of the properties of the material, such as structure, color and valuable substances like vitamins [1].

The morphological structure of the material is quite important, since it influences heat and mass transport, and thus the movement of the sublimation front [2]. As microstructural changes are a local phenomenon, which usually take place in the vicinity of the sublimation front, it is important to understand the structural formation during freeze-drying. One phenomenon is the collapse of the matrix, which can happen if the critical temperature is exceeded [3]. Due to the loss of product quality, this phenomenon must be prevented in common freeze-drying processes. Since in the past, such in-situ observations were missing, in industrial processes, large safety margins are usually applied to prevent product damage. By applying moderate drying conditions and prolonged freeze-drying times, the process time and energy costs are increasing. A valuable tool to observe microstructural changes during a drying process is $\mu-\mathrm{CT}$ measurement. Some scientific studies on the detection of these changes have recently been published. Siebert et al. [4] observed the crust formation during hot-air-drying. By drying carrot discs to different final relative moisture contents, they could show that with increasing drying time, the thickness of the crust increases. The same group also visualized the structure development during freeze-drying by using $\mu-C T$ measurement [5]. Here they used freeze-drying, microwave-drying and serial combinations of these two drying methods to show the impact of the drying process on the structural changes during the drying of carrot discs. Their main 
finding was that a radial drying front emerged. To prove their finding, they used a partly dried sample, and could show that the center of the carrot disc dried first. The center of the carrot disc showed a porous part, while the outer parts were dense and collapsed. A big disadvantage of their study was that they didn't use in-situ measurements; instead, the drying process was carried out externally.

Gruber et al. [2] highlighted the advantages of in-situ measurements during freeze-drying. By using neutron imaging, they were able to observe the movement of the sublimation front inside a particle bed. Here they used a custom-built freeze-drying cell filled with frozen maltodextrin particles. The main findings here were that the particle bed forms a planar moving sublimation front from the bottom to the top, and a radial drying front in each particle. These findings contrasted with simulations from literature [6-8], and show the necessity to carry out experimental studies on the visualization of the sublimation front. However, Gruber et al. [2] made their stage from aluminum, which is not suitable for $\mu$-CT measurements. Other in-situ measurements during freeze-drying were made by Nakagawa et al. [9]. Here they employed synchrotron radiation to observe structural formations in frozen and annealed dextrin solutions during freeze-drying. In their study, they used a freeze-drying stage that had a housing of acrylic resin connected to a vacuum pump. The heat was applied at the bottom of the stage by using a tempered air flow. However, they could not control the temperature exactly.

Altogether, the studies show the importance of using imaging techniques such as $\mu-C t$ measurements when studying freeze-drying. Gruber et al. [2] and Nakagawa et al. [9] could also prove the necessity of in-situ measurements. Therefore, it is the aim of this study to develop a freeze-drying stage for in-situ measurements in a $\mu$-CT. This stage will be made from a material that allows $\mathrm{X}$-rays to penetrate, while it is tight enough to apply a vacuum. The stage should also be fully controllable in respect of temperature and pressure.

\section{Approach and Development}

\section{1. $\mu-C T$ Unit}

The stage was basically developed for the $\mu$-CT XCT-1600 HR, built by Nordson Matrix Technologies GmbH, Feldkirchen, Germany, which is shown in Figure 1. With this current $\mu$-CT setup, it is possible to measure in a resolution range from $16 \mu \mathrm{m}$ to $1 \mu \mathrm{m}$ voxel size. This corresponds to a maximum sample diameter of $32 \mathrm{~mm}$ for a $16 \mu \mathrm{m}$ voxel size and $2 \mathrm{~mm}$ for a $1 \mu \mathrm{m}$ voxel size.

The XWT-160-TC transmission X-ray tube (X-RAY WorX GmbH, Garbsen, Germany) (Figure 1, Nr. 1), with up to $80 \mathrm{~W}$ emission power, provides a $10 \mathrm{~W}$ target power. Built-in is a high brightness target with a $250 \mu \mathrm{m}$ beryllium layer as a substrate, and a tungsten active layer of $6 \mu \mathrm{m}$ thickness. High resolutions are produced by the resulting cone beam, which enlarges the stated sample.

Figure 1, Nr. 2 shows the sample manipulator with the fixed freeze-drying stage. The manipulator can be moved in all three directions, while tomographic volumes are recorded, up and down, nearer or farther from the tube, as well as to the left and right. By rotating the built-in rotary table, multiple radiographic images at different scanning angles can be generated.

To measure the attenuated X-rays, a CCD-1100XR detector (VDS Vosskühler GmbH, Osnabrück, Germany) is used. It has an active area of $36.18 \mathrm{~mm}(\mathrm{H})$ by $24.12 \mathrm{~mm}(\mathrm{~V})$, and is subdivided in $4024(\mathrm{H})$ by $2680(\mathrm{~V})$ pixels at $3 \mathrm{fps}$. These pixels are then binned by a $2 \times 2$ pattern. Its $150 \mu \mathrm{m}$ scintillator is manufactured from CsI (Cesium-Iodide). The whole detector is shown in Figure 1, Nr. 3.

Number 4 in Figure 1 is the electrical, cooling and vacuum connection of the freeze-drying stage. Number 5 is a spring that assists the winding of the connection cables during the rotation. 


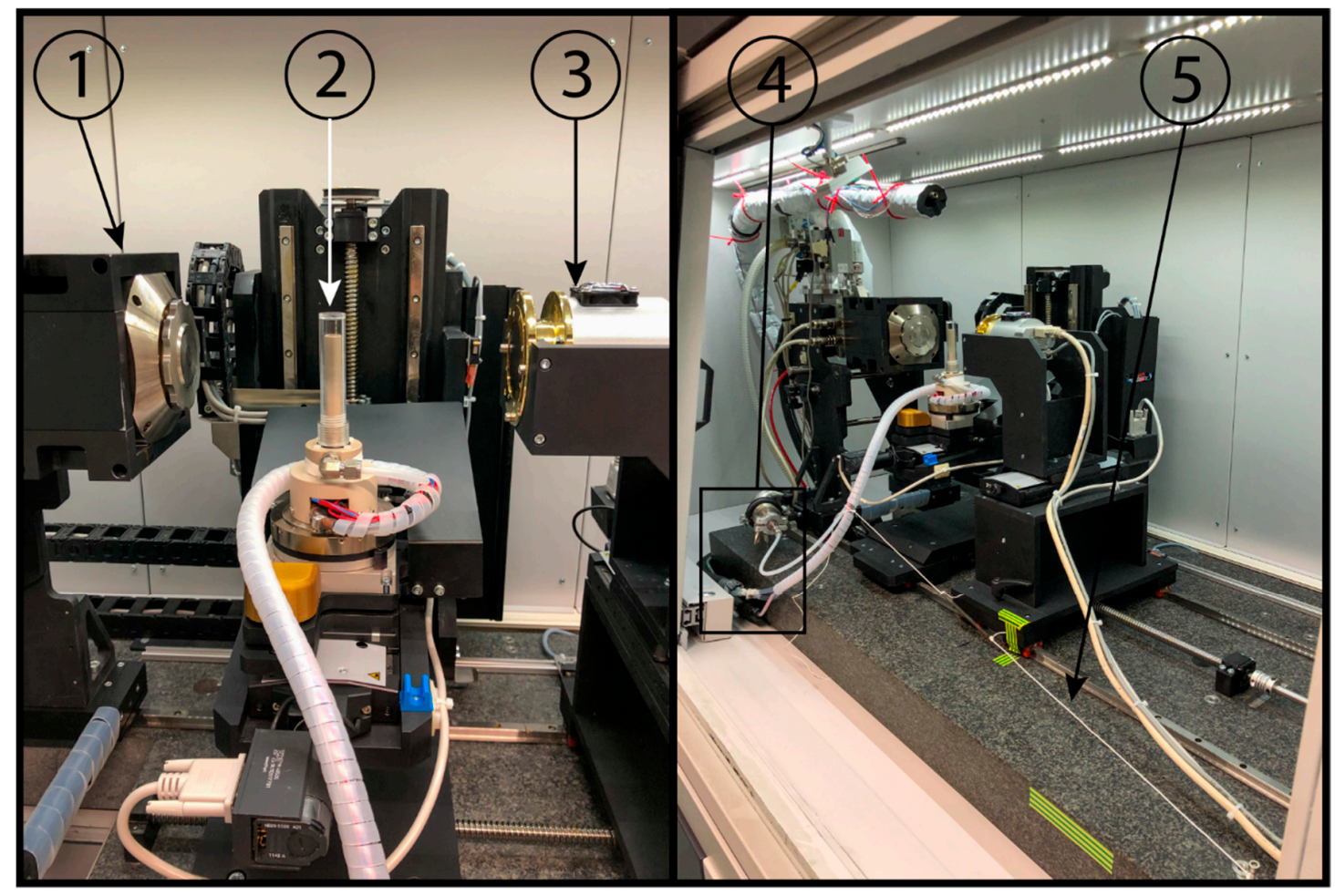

Figure 1. In-house XCT-1600 HR $\mu$-CT; (1) X-ray tube; (2) Fixed freeze-drying stage; (3) CCD-11000XR detector; (4) Stage-Connection; (5) Winding support.

\subsection{Development of the Freeze-Drying Unit}

The stage was developed considering the main features of freeze-drying and X-ray imaging: temperature, pressure, housing material and the required resolution. Preliminary tests with a scanning electron microscope showed that due to the minimal thickness of the lamellae between the pores, a minimal resolution of $1 \mu \mathrm{m}$ voxel size is necessary. As the main scope of the experiments is to do in-situ freeze-drying experiments and observe the process for maltodextrin and sucrose samples, it is necessary that the sample holder can be cooled to a minimum of $-40{ }^{\circ} \mathrm{C}$. As the glass transition temperature of the maximum freeze concentrated product is a critical temperature in freeze-drying processes, the chosen temperature allows a buffer to the glass transition temperature of $-32{ }^{\circ} \mathrm{C}$ for sucrose [10].

Secondly, the chamber must be airtight, for vacuums down to $10 \mathrm{~Pa}$. This pressure corresponds to a sublimation temperature of $-42{ }^{\circ} \mathrm{C}$ for water. In addition, it is necessary that the mechanical stability of the stage is high enough to withstand the vacuum.

In addition to the stability, a voxel size of $1 \mu \mathrm{m}$ is required. To get such high resolutions, the sample holder must be positioned close to the $\mathrm{X}$-ray tube. This results in the requirement of a very thin enclosure, which is, in the beam-area, as penetrable as possible for X-rays. Finally, the stage needs to fit into the manipulator of the $\mu$-CT unit.

To reach $-40{ }^{\circ} \mathrm{C}$, a three-stage thermoelectric cooler (uwe-electronic $\mathrm{GmbH}$, Unterhaching, Germany) (Figure 2, Nr. 9) is built in and controlled by a TEC-Controller (Head Electronics GmbH, Prien, Germany). The advantage of the three stages is that the thermoelectric cooler can manage high $\Delta \mathrm{T}$ between the hot and the cold side. However, a disadvantage is that it reaches low temperatures comparatively slowly. As freeze-drying is a slow process, this detriment can be neglected. The sample is cooled by discharging the heat through a copper bar (Figure 2, Nr. 2). By using the top of the copper bar as the sample holder, heat conduction is facilitated due to the high thermal conductivity of copper. To control the sample temperature with the thermoelectric cooler, a PT1000 sensor is inserted into a bore hole (Figure 2, Nr. 3) in the copper bar. A thermoelectric cooler produces heat on one 
side of the element. To reach low temperatures, it is essential to remove the produced heat. To do so, a custom-made copper heat sink (Figure 2, Nr. 10) is installed and fixed by screws in the main housing (Figure 2, Nr. 8). To remove the heat, it is connected to a heat exchanging device (Peter Huber Kältemaschinenbau AG, Offenburg, Germany), which is operated outside of the $\mu$-CT and rinses the heat sink with coolant (30\% ethylenglycol and 70\% demineralized water). Another advantage of the heat sink is that it is also the fixation of the thermoelectric cooler against the copper bar.

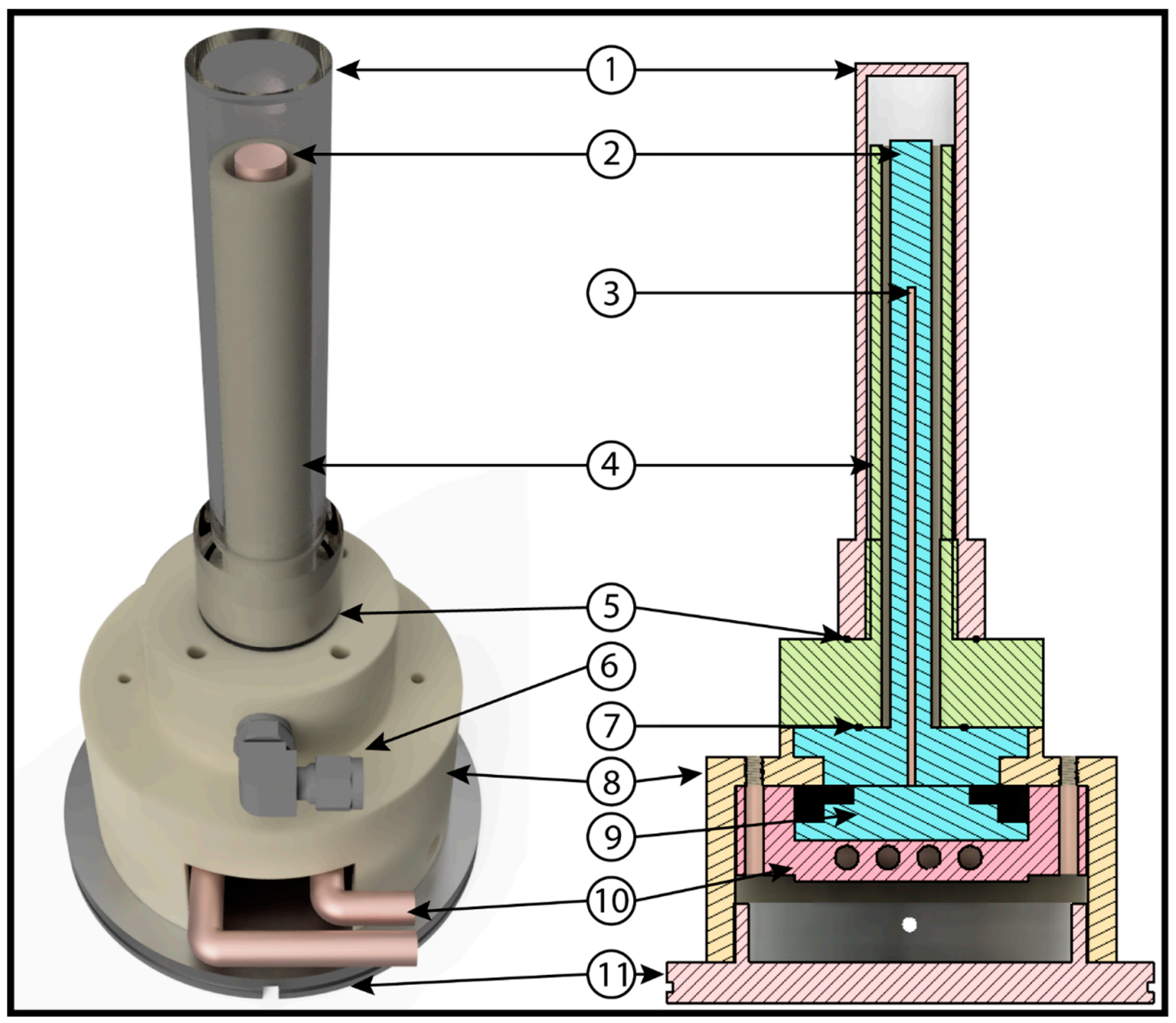

Figure 2. Isometric and sectional view of the developed freeze-drying stage for $\mu$-CT measurements; (1) cap; (2) sample holder; (3) PT1000 bore hole; (4) vacuum chamber with thread to mount the cap; (5) seal; (6) Swagelok connecter; (7) seal; (8) electrical housing; and (9) thermoelectric cooler; (10) heat sink; (11) support plate.

To provide the required vacuum of $10 \mathrm{~Pa}$, a sealed enclosure was developed. As can be seen in Figure 2, the enclosure consists of five different parts: the copper bar (Nr. 2); two sealing rings (Nr. 5 and 7); the actual vacuum chamber (Nr. 4); and the cap (Nr. 1). In order to facilitate the rotation for the tomographic measurements, as well as the reconstruction of the later volumes, all these parts were developed in a rotation-symmetric cylindrical shape. Furthermore, parts 1 and 4 , as well as the housing for the thermoelectric cooler (Nr. 8), are made of polyetheretherketon (PEEK). In Figure 2, the cap is visualized with an acrylic material. This cap was used for early stage experiments outside the $\mu-\mathrm{CT}$, and is used in this figure for visibility reasons. To make fast sample application possible, they are fitted onto the vacuum chamber by a great thread sealed by a sealing ring (Figure 2, Nr. 5).

The vacuum chamber (Figure 2, Nr. 4) is screwed onto the main housing by four polypropylen-6 screws, and sealed by another sealing ring (Figure 2, Nr. 7). The upper part of this component is a hollow cylinder which is used as additional isolation of the copper bar, and as a basis for the thread to 
connect the cap and the chamber. Due to the risk of attenuating the X-ray beam in front of the sample, the height of the hollow cylinder is slightly smaller than the copper bar. To evacuate the vacuum chamber, a 90-tube fitting (Figure 2, Nr. 6) (Swagelok Company, Solon, OH, USA) was installed and connected to a rotary vane pump (E2M1.5, Edwards, Feldkirchen, Germany), which was controlled by a CVC3000 controller, VV6 valve and a ventilation valve, and measured by a VSP3000 pirani sensor (Vacuubrand GmbH u. Co KG, Wertheim, Germany).

Since the thermoelectric cooler and its heat sink have to fit in the main housing, it is the widest part of the stage. The support plate (Figure 2, Nr. 11) was designed as an exchangeable mounting device. This was done to keep the stage adaptable for other $\mu$-CT units and experiments in an easy way. As the plate is fixed to the experiment's manipulator by screws, the plate is constructed out of stainless steel.

For drying experiments with solutions, a PEEK extension (Figure 3, right) with a height of $10 \mathrm{~mm}$ and a diameter equal to the sample holder was constructed. Due to the watertight connection, solutions can be filled in and frozen inside the $\mu$-CT unit.

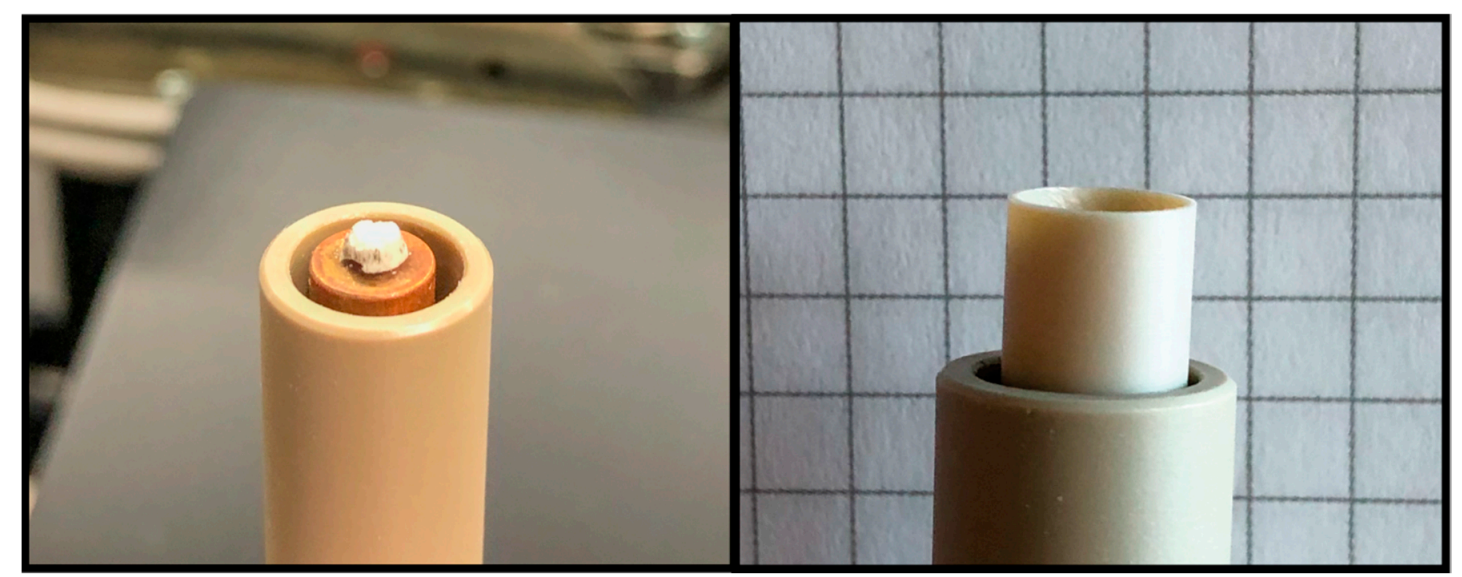

Figure 3. Left: Particle sample on the sample holder; Right: Cylindrical cup-attachment for packed beds and liquids.

\section{Experiments}

\subsection{Validation Experiments}

To prove the functionality of the developed freeze-drying stage, several experiments were made. The first drying experiments were carried out outside of the $\mu-\mathrm{CT}$ unit. They were made to check the basic functions as vacuum and temperature. The vacuum tightness was verified by evacuating the stage until the pressure was not decreasing anymore; this happened at a pressure of $7 \mathrm{~Pa}$. While the vacuum was not a problem, the required temperature of $-40{ }^{\circ} \mathrm{C}$ could not be reached by the first prototype. Two problems hindered the thermoelectric cooler to reach the selected temperature. First, the used heat exchanger (Deben UK Limited, Woolpit, UK) was not able to discharge the developed heat from the cooler. After changing the exchanger to a more powerful device, lower temperatures were possible, but the requested temperature still could not be reached. The main problem was that the former version was built out of stainless steel, and the heat conduction of the material was far too high. This was a result of the combination of the high heat conduction of the stage and temperatures of about $30^{\circ} \mathrm{C}$ inside the $\mu$-CT. After changing the material to PEEK, the expected temperature was reached.

PEEK is a very rigid high-performance synthetic material [11]. Due to its mechanical stability, it is suitable for the fabrication of the described stage. Furthermore, the low heat conduction and the fact that it is comparatively penetrable for X-ray beams made it a perfect material to set up the freeze-drying stage. PEEK can also be used in high-energy facilities such as a synchrotron. Therefore, the cap, which was originally made of acryl, was also changed to PEEK. 


\section{2. $D$ and $3 D$ Visualization of the Freeze-Drying Process}

\subsubsection{Freeze-Drying of Solutions}

For the experiments in the $\mu-C T$, different test samples were used. At first, experiments with $75 \mu \mathrm{L}$ liquid solutions of maltodextrin (Glucidex 12D, Roquette, Lestrem, France) or a sucrose (Carl Roth $\mathrm{GmbH}$ und Co. KG, Karlsruhe, Germany) solution of $5 \%$ or $20 \%(\mathrm{w} / \mathrm{w})$ were filled into the cup attachment (Figure 3, right), and then frozen inside the stage. To do so, the filled sample holder was cooled down to $-40{ }^{\circ} \mathrm{C}$ with a defined ramp, and then held for $20 \mathrm{~min}$. To reach that temperature, the heat exchanging device for counter-cooling was set to $-12{ }^{\circ} \mathrm{C}$.

The standard experimental procedure was to generate three tomographic volumes during one drying run: first of the frozen sample; then after half of the estimated drying time; and finally of the fully dried sample. In between, radiographic images were taken at defined time intervals, resulting in three 3D volumes of the sample and a 2D image sequence in which the drying process and changes in the sample structure could be observed. The drying process was started by lowering the pressure to $10 \mathrm{~Pa}$, which corresponds to the sublimation temperature of $-42{ }^{\circ} \mathrm{C}$ for water. Afterwards, the shelf temperature was raised to $-34{ }^{\circ} \mathrm{C}$ for sucrose and $-12{ }^{\circ} \mathrm{C}$ for maltodextrin. Both temperatures were selected to be as close as possible to the corresponding glass transition temperature of the maximum freeze-concentrated solution of the materials to reach the fastest drying time without risking structural collapse. To generate a tomographic volume, the drying process must be interrupted. This was done by increasing the pressure to atmospheric pressure, and lowering the temperature back to $-40{ }^{\circ} \mathrm{C}$. The sample remained in the $\mu$-CT until the completion of the experiment.

\subsubsection{Freeze-Drying of Frozen Particles}

Furthermore, experiments with single maltodextrin and sucrose particles with a diameter of about $3.5 \mathrm{~mm}$ and a solid content of $5 \%$ and $20 \%(\mathrm{w} / \mathrm{w})$ were carried out, respectively. The maltodextrin particles were produced by spraying the liquid solution with an ultrasonic nozzle (Sonaer Inc., New York, NY, USA) into liquid nitrogen. The ultrasound was switched off, and the flow rate was set to $2.5 \mathrm{~mL} / \mathrm{min}$. The sucrose particles were produced by dropping single droplets with a syringe (Braun, Sterican, $0.80 \times 50 \mathrm{~mm} \mathrm{BL} / \mathrm{LB}$ ), with an inner diameter of $0.8 \mathrm{~mm}$, into liquid nitrogen. Until usage, the particles were stored in a $-80^{\circ} \mathrm{C}$ freezer. To facilitate the sample handling, only hemispheric particles were used for the freeze-drying experiments. They were formed during the production process beside the typical spherical particles. Before starting the experiment, the thermoelectric cooler was set to $-40{ }^{\circ} \mathrm{C}$, and the sample then positioned on the sample holder. After sealing the cap, the same procedure as in the previous experiments was performed.

\subsection{Image Acquisition and Processing}

All experiments were performed with a resolution of $3 \mu \mathrm{m}$ voxel size and an acceleration voltage of $60 \mathrm{kV}$. Before every experiment, a gray value adjustment was performed to eliminate the cap and pixel failures in the projections. The adjustment is an algorithm that begins by taking a dark field image and subtracting it from the raw image. The resulting image is then adjusted by a flatfield correction, resulting in a final image where mainly the sample is visible.

The exposure time varied between the experiments, ranging from 1796 to $1937 \mathrm{~ms}$. For each tomographic volume, 2000 projections-except for the sucrose solution with 2500 projections-from different angles within a $360^{\circ}$ turn were made, and later reconstructed. To improve the contrast of the tomographic volumes, the integration function was used in two experiments. This tool takes more than one image per projection and calculates the average of the generated images, resulting in a better signal-to-noise ratio, thus resulting in the better visibility of the sample [12].

Radiographic image sequences were inverted, and the contrast automatically adapted with ImageJ v1.51s (National Institute of Health, Bethesda, MD, USA). Image reconstruction to a volume of $2000 \times 2000 \times 1225$ voxel was performed using MIPS-CT v5.1.6.0 (Nordson Matrix Technologies 
$\mathrm{GmbH}$, Feldkirchen, Germany), and visualized by CERA Xplorers v5.1.0 (Siemens Healthcare GmbH, Erlangen, Germany). The used FDK reconstruction algorithm is a backprojection-based algorithm, which is described in detail in Feldkamp et al. [13]. To generate an easily interpretable image, the figures shown in this work were modified with a size 5 median filter (MAVI v1.5.2, Fraunhofer ITWM, Kaiserslautern, Germany) [14].

\section{Results}

The experiments showed good results, and proved the functionality of the stage to perform in-situ freeze-drying runs. This chapter shows examples of some of the results which underline the benefits and the functionality of the stage.

Both images in Figure 4 show the same 20\% (w/w) maltodextrin particle from the same angle at different drying times. The left image shows the particle after half of the estimated drying time of $60 \mathrm{~min}$. Whilst the dark gray represents the already dried part, the bright gray/white is the still-frozen area of the particle. The particle on the right image is fully dried. Here, the gray sectors represent the sugar lamella, while the black areas are pores. Due to overheating, after the left tomographic volume was taken, the inner structure collapsed. The dark areas indicated by arrows in the core are large vertical pores which developed during the meltdown. The brighter lines where more radiation is attenuated-especially in the particles core and on the left edge-indicate regions where the sugar is concentrated. How the concentration of sugar is developing during the collapse and drying process, respectively, were not examined in this study.

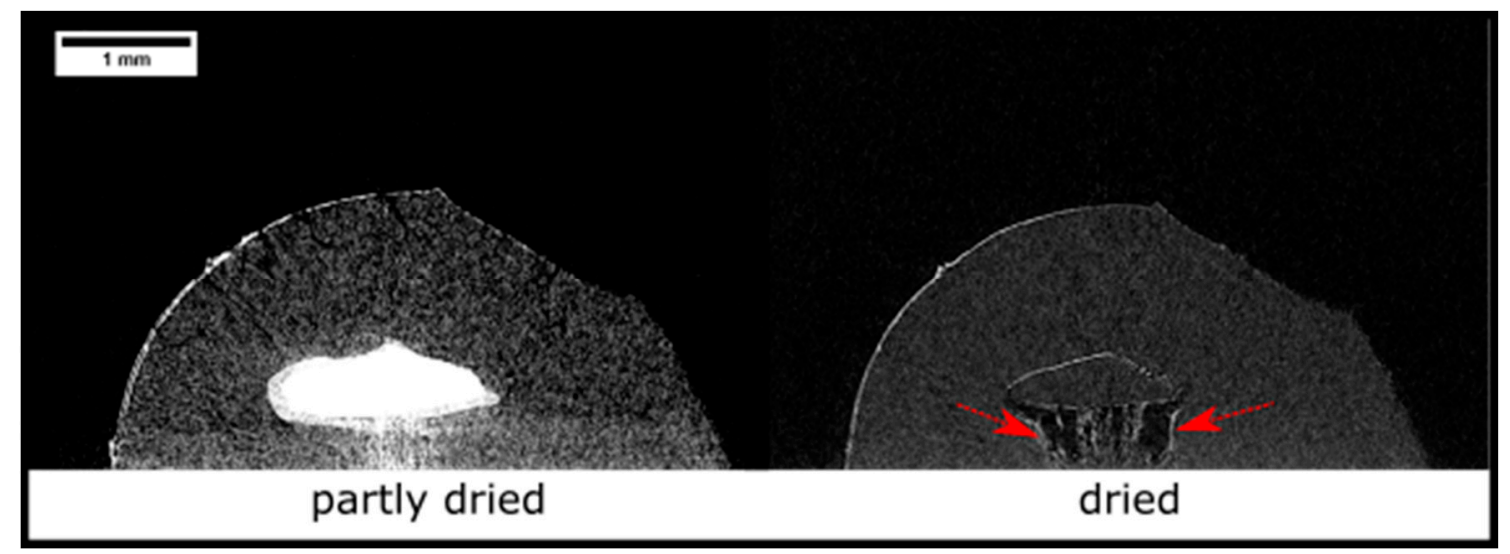

Figure 4. Partly dried and dried sectional view of a 20\% (w/w) maltodextrin particle. Before (Left) and after (Right) a structural collapse.

However, the dried microstructure of the particle is visible, and its changes during the drying process can be observed. Furthermore, the drying kinetics can be recorded by radiographic imaging.

The emerging image sequence shows the exact movement of the drying front, as well as the evolving structure. The given image sequence in the online Supplementary Materials, Video S1, shows a time lapse of a $20 \%(\mathrm{w} / \mathrm{w})$ sucrose solution. The sample was measured with a resolution of $3 \mu \mathrm{m}$ voxel size, and every $48 \mathrm{~s}$ a radiographic image was taken. Here, the bright gray parts represent the ice in the sample, while the dark sectors are already dried.

Drying and measuring sucrose particles showed that their absorption of X-rays is higher than maltodextrin. Figure 5 shows that the pore structure of the dried $20 \%$ w/w) sucrose particle is clearly visible. Apart from the different absorption, it also seems that the pore walls of the inner structure are thicker and the pores bigger than the ones in Figure 4. For the acquisition of this volume, the integration function was used. For every projection, six images were taken. This function, as well as the reason that the attenuation coefficient for sucrose is nearly four times higher than for maltodextrin, is probably responsible for the better visibility [15]. 


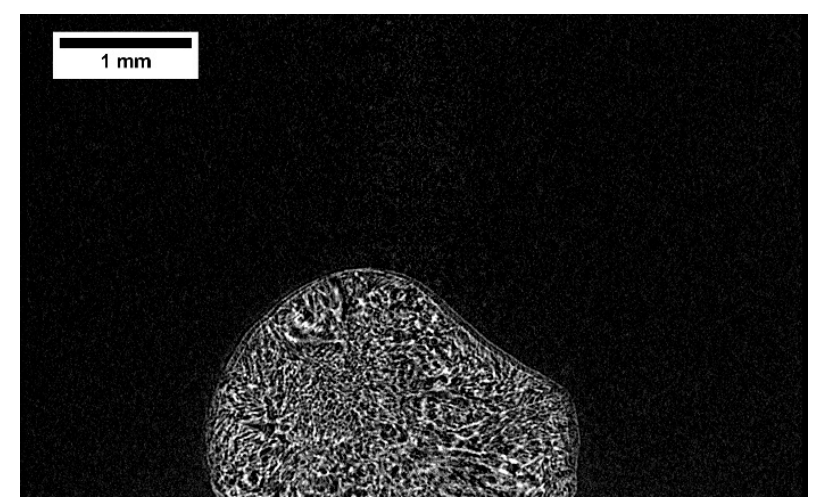

Figure 5. Sectional view of a dried $20 \%(w / w)$ sucrose particle.

Freeze-drying of solutions, mostly executed in the pharmaceutical industry, was also performed in this work. Therefore, the cylindrical extension was filled with $5 \%(\mathrm{w} / \mathrm{w})$ sucrose solutions. A sectional view of the reconstructed volume is shown in Figure 6; the solid structure is clearly visible. It can be observed that the larger pores in the dried sample mainly evolve in a vertical direction. Freezing the solution inside the stage with determined ramps creates defined conditions which allow the repetition of the experiments under the same conditions. Compared to Figure 5, the contrast is slightly worse, as this volume was only generated with three pictures per projection. In addition, the total solid is $5 \%(w / w)$ lower than the total solid of the sample in Figure 5, and so the total attenuation is also lower.

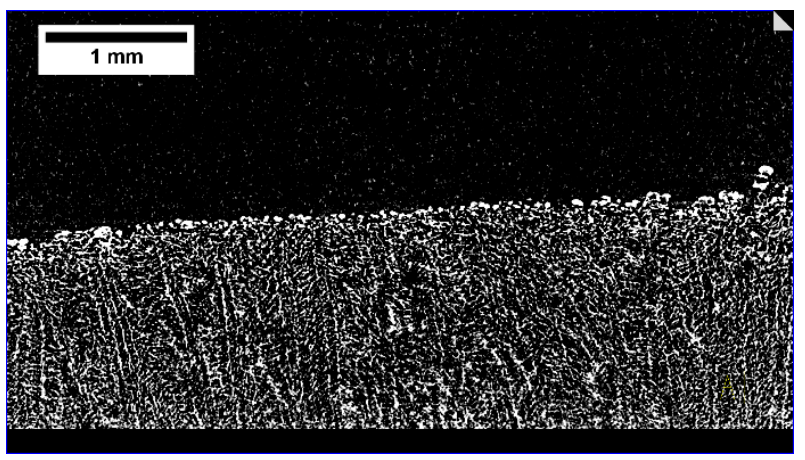

Figure 6. Sectional view of a dried $5 \%(\mathrm{w} / \mathrm{w})$ sucrose solution.

The performed experiments show the great potential of the stage for other applications that require a controlled environment such as heating, moisture adsorption or convective drying. Not only the structure of samples is visible, but the drying kinetics is also traceable. In addition, the polarity of the thermoelectric cooler can be changed and used to heat the sample holder. This modification results in a stage where many different experiments, which require a controlled environment, can be performed.

The right 3D volume slice in Figure 7 shows a partly dried sample of an egg white protein particle. The frozen particle was produced in the same way as the sucrose particles. The core part of the sample is still frozen, while the border is already dried. This experiment shows that the imaging process is also suitable for more complex systems.

The left 2D image pictures the beginning of the coagulation of egg white protein. This image is the result of a heating experiment with the stage. The bright parts are already coagulated, and the dark circles correspond to air bubbles. For this experiment, raw egg white protein was heated at atmospheric pressure to $70{ }^{\circ} \mathrm{C}$ with $0.2{ }^{\circ} \mathrm{C} / \mathrm{s}$, and the coagulation process was observed. During the process, the movement of the bubbles and the coagulation itself were visible.

Due to the modularity of the stage, different caps can be developed for other fields of use. By changing the material, the stage could also be used for further experiments with neutron imaging. 


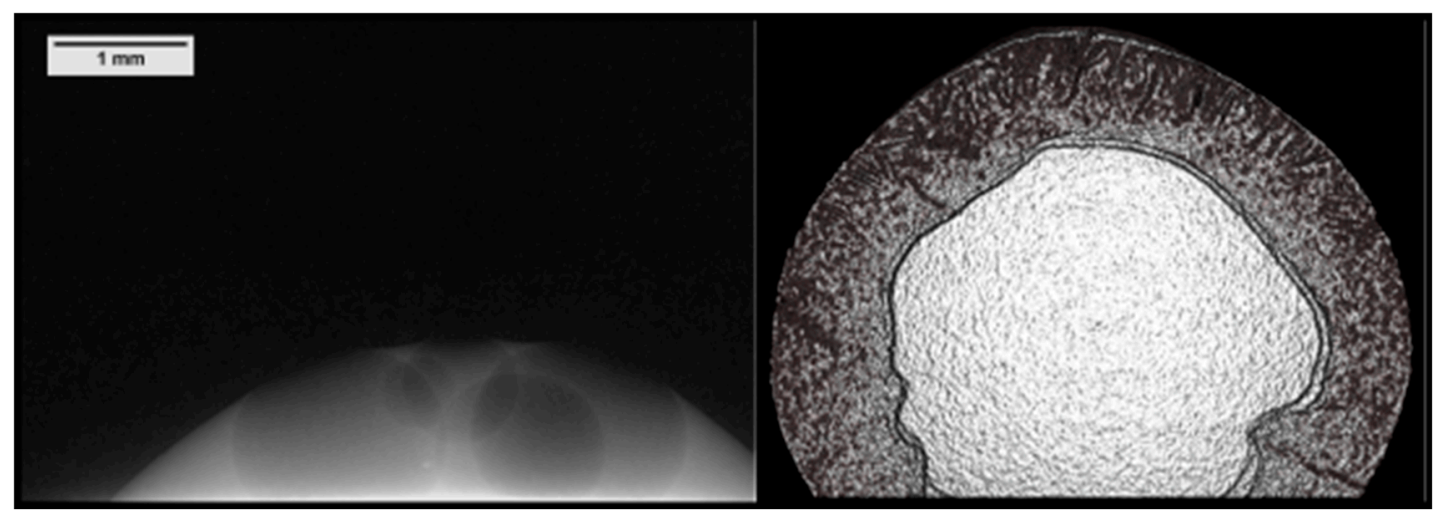

Figure 7. Left: Coagulation of chicken protein (radiographic image); Right: Slice of a partly dried chicken protein particle.

\section{Conclusions and Outlook}

This work shows the development of a freeze-drying stage for $\mu$-CT measurements. With this stage, it is possible to study the freeze-drying properties of a single particle, particle beds or solution - such as the evolution of pore structure-in more detail. Particle beds or solutions can be studied by implementing a cylindrical cup attachment made of PEEK. In particular, the observation of the freeze-drying properties of solutions can be studied. As the solution is directly frozen inside the stage, we can study all process steps of the freeze-drying process in full detail. By the adaptable design of the stage, we will be able to use this freeze-drying stage for not only $\mu$-CT measurements. The shape and the material of the parts that come into contact with the beam are easy to modify for other uses such as synchrotron or neutron imaging. This will allow us an even better investigation of the freeze-drying process, since we will benefit from the complementary information of both imaging methods [16]. By enabling defined sample environments, we are not only able to study the freeze-drying process, but also other processes that require a controlled environment, such as convective drying.

Overall, the developed freeze-drying stage for in-situ measurements gives us a valuable tool to study structural changes during freeze-drying in more detail. In the future, an image processing tool will be developed by us in cooperation with researchers from Otto-von-Guericke University Magdeburg, Germany (OVGU), which will allow us to quantitatively examine the freeze-drying process in more detail. These findings will help us to develop a pore network model together with the OVGU.

Supplementary Materials: The following is available online at http://www.mdpi.com/2227-9717/8/7/869/s1. Video S1: In-situ freeze-drying process of a $20 \%(\mathrm{w} / \mathrm{w})$ sucrose solution, visualized by X-ray radiography with a $3 \mu \mathrm{m}$ voxel size.

Author Contributions: Conceptualization, M.H. and S.G.; Investigation, M.H.; Project administration, P.F.; Supervision, S.G.; Visualization, M.H.; Writing—original draft, M.H.; Writing—review \& editing, M.H., S.G. and P.F. All authors have read and agreed to the published version of the manuscript.

Funding: This research made use of the $\mu-\mathrm{CT}$ funded by the Deutsche Forschungsgemeinschaft (DFG INST95/1111-1). Furthermore, it also made use of further equipment that was funded by the Deutsche Forschungsgemeinschaft: 198187031. The authors thank the Deutsche Forschungsgemeinschaft for their financial support for this work within the Fo 357/5-1.

Conflicts of Interest: The authors declare no conflict of interest.

\section{References}

1. Liapis, A.; Bruttini, R. Freeze Drying. In Handbook of Industrial Drying, 4th ed.; Mujumdar, A., Ed.; CRC Press Inc.: Boca Raton, FL, USA, 2014. 
2. Gruber, S.; Vorhauer, N.; Schulz, M.; Hilmer, M.; Peters, J.; Tsotsas, E.; Foerst, P. Estimation of the local sublimation front velocities from neutron radiography and tomography of particulate matter. Chem. Eng. Sci. 2020, 211, 115268. [CrossRef]

3. Pikal, M.J.; Shah, S.; Senior, D.; Lang, J.E. Physical chemistry of freeze-drying: Measurement of sublimation rates for frozen aqueous solutions by a microbalance technique. J. Pharm. Sci. 1983, 72, 635-650. [CrossRef] [PubMed]

4. Siebert, T.; Zuber, M.; Engelhardt, S.; Baumbach, T.; Karbstein, H.P.; Gaukel, V. Visualization of crust formation during hot-air-drying via micro-CT. Dry. Technol. 2019, 37, 1881-1890. [CrossRef]

5. Siebert, T.; Zuber, M.; Hamann, E.; Baumbach, T.; Karbstein, H.P.; Gaukel, V. Micro-CT visualization of structure development during freeze-drying processes. Dry. Technol. 2019, 11, 1-9. [CrossRef]

6. Trelea, I.C.; Passot, S.; Marin, M.; Fonseca, F. Model for heat and mass transfer in freeze-drying of pellets. J. Biomech. Eng. 2009, 131, 74501. [CrossRef] [PubMed]

7. Chitu, T.; Vessot, S.; Peczalski, R.; Andrieu, J.; Woinet, B.; Françon, A. Influence of Operating Conditions on the Freeze-Drying of Frozen Particles in a Fixed Bed and Modeling Data. Dry. Technol. 2015, 33, 1892-1898. [CrossRef]

8. Liapis, A.I.; Bruttini, R. A mathematical model for the spray freeze drying process: The drying of frozen particles in trays and in vials on trays. Int. J. Heat Mass Transf. 2009, 52, 100-111. [CrossRef]

9. Nakagawa, K.; Tamiya, S.; Sakamoto, S.; Do, G.; Kono, S. Observation of Microstructure Formation During Freeze-Drying of Dextrin Solution by in-situ X-ray Computed Tomography. Front. Chem. 2018, 6, 418. [CrossRef] [PubMed]

10. Slade, L.; Levine, H. Non-equilibrium behavior of small carbohydrate-water systems. Pure Appl. Chem. 1988, 60, 1841-1864. [CrossRef]

11. Kurtz, S.M. Chemical and Radiation Stability of PEEK. In PEEK Biomaterials Handbook; Elsevier: Amsterdam, The Netherlands, 2012; pp. 75-79.

12. Hendee, W.R.; Ritenour, E.R. Medical Imaging Physics, 4th ed.; Wiley-Liss: New York, NY, USA, 2010.

13. Feldkamp, L.A.; Davis, L.C.; Kress, J.W. Practical cone-beam algorithm. J. Opt. Soc. Am. A 1984, 1, 612. [CrossRef]

14. Tan, L.; Jiang, J. Image Processing Basics. In Digital Signal Processing: Fundamentals and Applications; Tan, L., Jiang, J., Eds.; Elsevier: Amsterdam, The Netherlands, 2019; pp. 649-726.

15. Chantler, C.T. Detailed Tabulation of Atomic Form Factors, Photoelectric Absorption and Scattering Cross Section, and Mass Attenuation Coefficients in the Vicinity of Absorption Edges in the Soft X-Ray ( $Z=30-36$, $\mathrm{Z}=60-89, \mathrm{E}=0.1 \mathrm{keV}-10 \mathrm{keV})$, Addressing Convergence Issues of Earlier Work. J. Phys. Chem. Ref. Data 2000, 29, 597-1056. [CrossRef]

16. Schillinger, B.; Calzada, E.; Mühlbauer, M.; Schulz, M. Neutronenbilder zeigen was Röntgenstrahlen nicht sehen können. In Proceedings of the ICT 2008 2nd Conference on Industrial Computed Tomography (ICT), Wels, Austria, 26-28 February 2008.

(C) 2020 by the authors. Licensee MDPI, Basel, Switzerland. This article is an open access article distributed under the terms and conditions of the Creative Commons Attribution (CC BY) license (http://creativecommons.org/licenses/by/4.0/). 